University of Wollongong

Research Online

Australian Institute for Innovative Materials -

Papers

Australian Institute for Innovative Materials

$1-1-2018$

Twist-coil coupling fibres for high stroke tensile artificial muscles

Shazed Md Aziz

University of Wollongong, shazed@uow.edu.au

Sina Naficy

University of Sydney, snaficy@uow.edu.au

Javad Foroughi

University of Wollongong, foroughi@uow.edu.au

Hugh Ralph Brown

University of Wollongong, hbrown@uow.edu.au

Geoffrey M. Spinks

University of Wollongong, gspinks@uow.edu.au

Follow this and additional works at: https://ro.uow.edu.au/aiimpapers

Part of the Engineering Commons, and the Physical Sciences and Mathematics Commons

Research Online is the open access institutional repository for the University of Wollongong. For further information contact the UOW Library: research-pubs@uow.edu.au 


\title{
Twist-coil coupling fibres for high stroke tensile artificial muscles
}

\author{
Abstract \\ A new concept for tensile artificial muscles is introduced in which the torsional actuation of a twisted \\ polymer fibre drives a twist to writhe conversion in a serially attached elastomeric fibre. Thermally \\ induced torsional rotation of the twisted fibre caused formation of coils in the elastomeric fibre which \\ resulted in overall muscle length contraction. Theoretical predictions of the muscle strain were developed \\ by means of a modified single-helix theory. Experimental tests were conducted to measure the isotonic \\ contraction strains for elastomeric fibres of different diameters and lengths. A good agreement between \\ the measured and calculated results was found. Practical applicability of this muscle is evaluated by \\ using different mechanical loading conditions. Actuation contraction strains as high as $10 \%$ were \\ observed with excellent reversibility. Unlike original coiled fibre tensile actuators, these twist-coil artificial \\ muscles did not require any pre-conditioning cycles.

\section{Disciplines} \\ Engineering | Physical Sciences and Mathematics \\ Publication Details \\ Aziz, S., Naficy, S., Foroughi, J., Brown, H. R. \& Spinks, G. M. (2018). Twist-coil coupling fibres for high \\ stroke tensile artificial muscles. Sensors and Actuators A: Physical, 283 98-106.
}




\title{
Twist-Coil Coupling Fibres for High Stroke Tensile Artificial Muscles
}

\author{
Shazed Aziz ${ }^{1}$, Sina Naficy ${ }^{2}$, Javad Foroughi $^{1}$, Hugh R. Brown ${ }^{1}$, Geoffrey M. Spinks ${ }^{1 *}$ \\ ${ }^{1}$ ARC Centre of Excellence for Electromaterials Science and Intelligent Polymer Research \\ Institute, University of Wollongong, Innovation Campus, Squires Way, North Wollongong, \\ NSW, 2522, Australia. \\ ${ }^{2}$ School of Chemical and Biomolecular Engineering, The University of Sydney, Sydney, \\ NSW, 2006, Australia \\ *Correspondence to: Geoffrey M. Spinks (E-mail: gspinks@uow.edu.au)
}

\begin{abstract}
A new concept for tensile artificial muscles is introduced in which the torsional actuation of a twisted polymer fibre drives a twist to writhe conversion in a serially attached elastomeric fibre. Thermally induced torsional rotation of the twisted fibre caused formation of coils in the elastomeric fibre which resulted in overall muscle length contraction. Theoretical predictions of the muscle strain were developed by means of a modified single-helix theory. Experimental tests were conducted to measure the isotonic contraction strains for elastomeric fibres of different diameters and lengths. A good agreement between the measured and calculated results was found. Practical applicability of this muscle is evaluated by using different mechanical loading conditions. Actuation contraction strains as high as $10 \%$ were observed with excellent reversibility. Unlike original coiled fibre tensile actuators, these twist-coil artificial muscles did not require any pre-conditioning cycles.
\end{abstract}

Keywords: Artificial muscle, polymer fibre, twist-coil coupling, torsional actuator, tensile actuator 

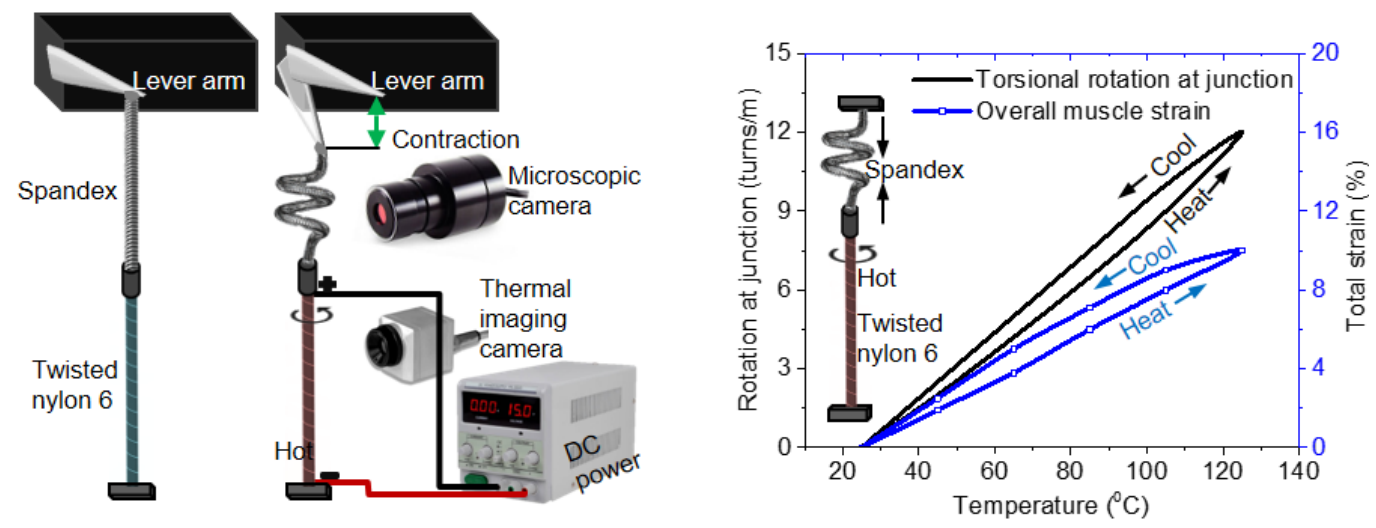

\section{Graphical Abstract}

\section{Introduction}

The idea of an artificial muscle dates back several decades with many materials now demonstrating volume and / or shape changes when stimulated [1-4]. General advantages of artificial muscles include their noiseless operation and capacity for miniaturisation without loss in power output per weight [5]. Artificial muscles have also been reported with three primary types of actuation: bending [6, 7], tensile [8, 9] and torsional [8, 10, 11]. Diverse applications of these muscles have been proposed ranging from soft robotics [12], prosthetic limbs [13], smart textiles [8, 9], exoskeletons [14], miniature fluidic mixers [2, 8], photonic displays [15], and energy harvesting devices [10].

Torsional artificial muscles made from twisted polymer fibres have created much interest, mainly because they are the basis for high performance tensile actuation when the twisted fibres are formed into helical coils [8, 10, 16-19]. Torsional actuation can be induced in twisted fibres by a volume change, such as thermal expansion. The thermally induced untwisting of drawn and twisted semicrystalline polymer fibres can be explained based on their anisotropic thermal properties where the untwisted fibres show comparatively large diameter expansion and negligible length direction change due to the high stiffness along the polymer chain axis. Twisting of these fibres results in helically oriented chains so that volume increases are accommodated by a partial fibre untwist. Thermal expansion and associated torsional actuation of twisted nylon- 6 fibres have been successfully modelled using a single helix geometry with the assumption that the polymer chains are inextensible [20]. In the systems where twisted fibres are mechanically translated to coiled structures, the 
torsion that accompanies volume expansion of the twisted fibre is translated into a length change in the coil. Tensile strokes as high as 50\% have been observed [8].

Here, we report a new mechanism of torsion driven tensile actuation, which is schematically illustrated in Fig. 1. A torsionally active non-coiled fibre is coupled in series with a second fibre that has a low critical torque for twist-induced coil formation. Significant overall length contraction occurs as coil turns form in the latter fibre due to the rotation of the torsionally active fibre. This effect can be optimised by adjusting the relative lengths of the fibres with respect to each other.

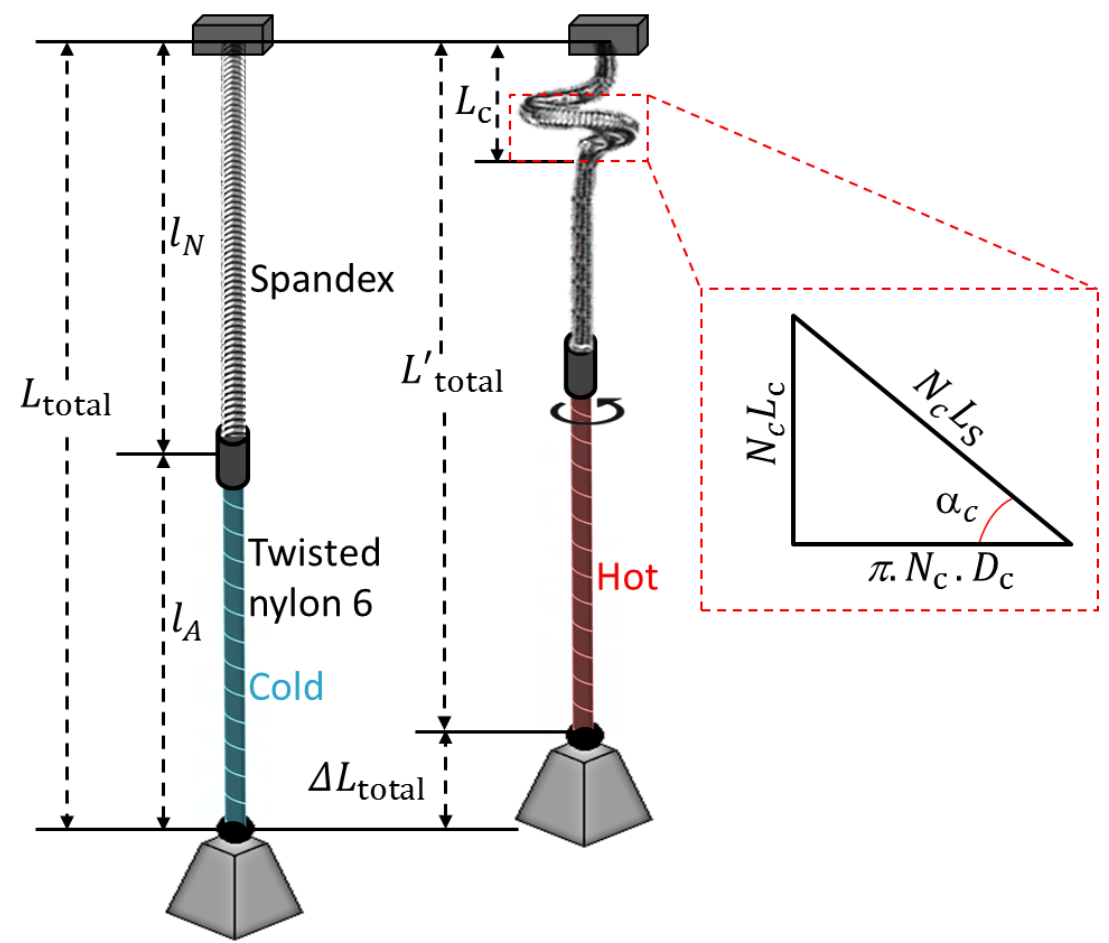

Fig. 1. Schematic illustration of the twist-coil actuator system: a torsionally active fibre (twisted nylon 6) is heated to cause rotation at the junction with a serially connected second fibre (Spandex). This rotation results in coil turn formation and linear, tensile contraction. The geometry of the coil turns is illustrated as an opened-up single-helix to show the relationships between torsional rotation and tensile contraction.

The overall length contraction $\left(\Delta L_{\text {total }}\right)$ of the twist-coil actuator is the summation of length changes in the elastomeric fibre (due to coil formation) and the twisted fibre (due to untwisting). In the former case, the length change as a result of coil formation can be predicted using the geometry of a single helix. Fig. 1 shows the schematic illustration of the twist-coil actuator arrangement. The total length before stimulation $\left(L_{\text {total }}\right)$ comprised the 
elastomer fibre length $\left(l_{\mathrm{N}}\right)$ and the length of the torsionally-active twisted polymer fibre $\left(l_{\mathrm{A}}\right)$. For each coil turn that forms, a straight segment of elastomeric fibre of length $L_{\mathrm{S}}$ is converted to a coil of length $L_{\mathrm{c}}$. Assuming that the contour lengths of both the elastomeric fibre and the twisted polymer fibre remain unchanged, then the formation of $N_{\mathrm{c}}$ coil turns in the elastomeric fibre causes an overall length change given by:

$$
\Delta L_{\text {total }}=N_{\mathrm{c}}\left(L_{\mathrm{c}}-L_{\mathrm{s}}\right)=N_{\mathrm{c}}\left(\pi D_{\mathrm{c}} \tan \alpha_{\mathrm{c}}-\frac{\pi D_{\mathrm{c}}}{\cos \alpha_{\mathrm{c}}}\right)=\pi N_{\mathrm{c}} D_{\mathrm{c}} \frac{\left(\sin \alpha_{\mathrm{c}}-1\right)}{\cos \alpha_{\mathrm{c}}}
$$

where $D_{\mathrm{c}}$ is the coil diameter and $\alpha_{\mathrm{c}}$ is the coil bias angle taken against the perpendicular direction to the long axis of the elastomeric return spring fibre. Here, $\Delta L_{\text {total }}$ is the contraction of the elastomeric fibre which also represents the overall tensile stroke of the series muscle since the length change during torsional actuation of the twisted fibre has been found to be negligible in previous studies [20].

Twist induced coil formation in fibres, wires and cables have been investigated using torsion mechanics $[21,22]$. Here, the critical torque $\left(\tau_{c}\right)$ needed to initiate coiling in the second fibre was estimated based on the criterion of Ross [23]:

$\tau_{\mathrm{c}}=\sqrt{2 E I F}$

where $E$ is the tensile modulus, $I$ is the second moment of area of the coiling fibre and $F$ is the applied tensile force. For fibres of circular cross-section and diameter $D_{\mathrm{y}}$, the second moment of area is $\pi D_{\mathrm{y}}{ }^{4} / 64$. Based on equation 2, coil formation is favoured in thin fibres of low elastic modulus. For this reason, a soft elastomeric fibre (commercially-available Spandex fibre) was used in the current work as the tensile actuator. The torsion was induced by heating a twisted and annealed nylon 6 fibre, as described previously [24]. The free torsional stroke per fibre length $\left(\Delta T_{\text {free }}\right)$ and generated blocked torque $\left(\tau_{\text {blocked }}\right)$ of a twisted fibre when stimulated can be described by [24]:

$$
\begin{aligned}
& \Delta T_{\text {free }}=\frac{n_{\mathrm{o}}}{l_{\mathrm{A}}}\left(\frac{d_{\mathrm{o}}}{d}-1\right) \\
& \tau_{\text {blocked }}=\Delta T_{\text {free }} \times J_{\mathrm{A}} G_{\mathrm{A}}
\end{aligned}
$$

where $n_{\mathrm{o}}$ is the initially inserted twist (turns) in the fibre having an initial length $l_{\mathrm{A}} ; d_{\mathrm{o}}$ and $d$ are the fibre diameters before and after heating; and $J_{\mathrm{A}} G_{\mathrm{A}}$ is the torsional modulus of the twisted fibre after heating; $G_{\mathrm{A}}$ is the fibre shear modulus after heating and $J_{\mathrm{A}}$ is the polar 
moment of area which is $\pi d^{4} / 32$ for fibres of circular cross-section. Equation 3 predicts the 'free', or unimpeded, torsional stroke. However, the attached Spandex fibre acts as a return spring (torsional modulus, $J_{\mathrm{N}} G_{\mathrm{N}}$; length, $l_{\mathrm{N}}$ ) as described previously $[2,24]$ and reduces the torsional stroke of the twisted fibre:

$\Delta T=\Delta T_{\text {free }}\left(\frac{J_{\mathrm{A}} G_{\mathrm{A}}}{J_{\mathrm{A}} G_{\mathrm{A}}+\frac{l_{\mathrm{A}}}{l_{\mathrm{N}}}\left(J_{\mathrm{N}} G_{\mathrm{N}}\right)}\right)$

The full range of generated torsional strokes and torques can be represented by the torque stroke curve as shown schematically as line A in Fig. 2. The effect of a return spring fibre is illustrated by the dashed line B having a slope equivalent to the torsional stiffness of the return spring. The intersection of lines $\mathrm{A}$ and $\mathrm{B}$ represent the final torsional stroke and residual torque applied by the torsional actuator on the return spring. The case of a return spring fibre that forms a coil when connected in series to a torsional actuator is represented by line $\mathrm{C}$. For clarity of presentation, this return spring is shown with a lower stiffness than the non-coiling return spring represented by line B. When the first coil appears, the torsional stiffness of the coiling return spring decreases as shown by the discontinuous slope of line $\mathrm{C}$. This first coil turn forms when the torque acting on the return spring reaches the critical torque $\left(\tau_{\mathrm{c}}\right)$ or equivalently the critical twist $\left(l_{\mathrm{N}} T_{\mathrm{c}}\right)$ inserted in the return spring fibre. The number of turns formed in the coil $\left(N_{\mathrm{c}}\right)$ depends on the remaining torsional stroke after the initiation of the first coil formation, so that:

$N_{\mathrm{c}}=\left(l_{\mathrm{A}} \Delta T-l_{\mathrm{N}} T_{\mathrm{c}}\right)$

Combining equations (1) and (6) gives the final predicted tensile stroke for the series actuator:

$\Delta L_{\text {total }}=\pi D_{\mathrm{c}}\left(l_{\mathrm{A}} \Delta T-l_{\mathrm{N}} T_{\mathrm{c}}\right) \frac{\left(\sin \alpha_{\mathrm{c}}-1\right)}{\cos \alpha_{\mathrm{c}}}$ 


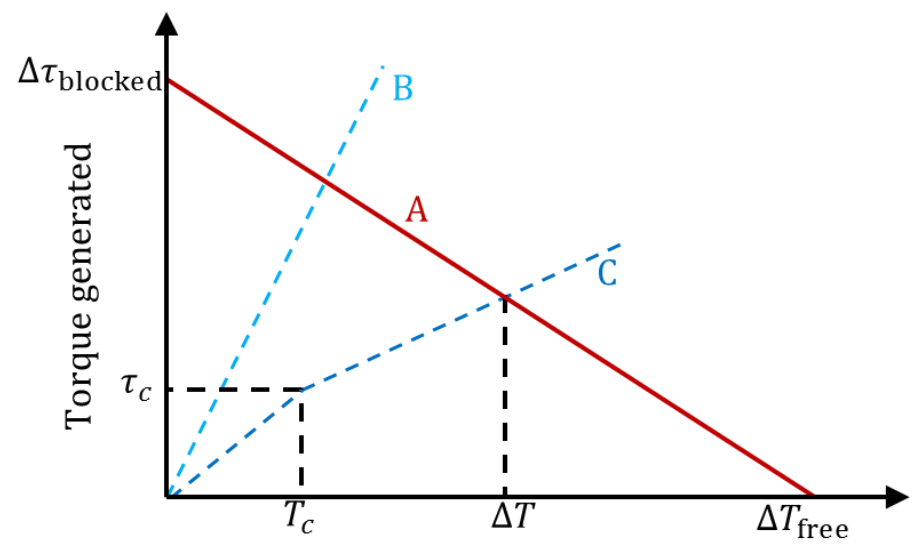

Torsional stroke generated

Fig. 2. Schematic illustration of the mix of torque and torsional stroke generated by a torsional actuator when activated (line A). The final torque and stroke generated depends on the external loading conditions. Free rotation generates the largest torsional stroke $\left(\Delta \boldsymbol{T}_{\text {free }}\right)$ and blocked rotation generates the largest torque $\left(\Delta \boldsymbol{\tau}_{\text {blocked }}\right)$. The torsional properties of noncoiling and coiling return spring fibres are represented by lines B and C, respectively.

A series of experiments were conducted using different lengths of Spandex fibre return spring and nylon 6 torsionally active fibres to evaluate equation (7). The length of each fibre affects the expected torsional stroke as described in equation (4): the torsional stiffness of the fibres depends inversely on fibre length, and the torsional free stroke of the actuating fibre is proportional to actuating fibre length. The tensile stroke is expressed as a percentage of the initial unloaded length of the series actuator to effectively compare the performance of different length combinations. The effect of the applied external load on coil formation and tensile stroke is also considered.

\section{Experimental}

\subsection{Materials}

Commercially sourced nylon 6 fibre $(0.54 \mathrm{~mm}$ diameter, Sport Fisher monofilament fishing line), silver coated nylon multifilament fibre $(0.50 \mathrm{~mm}$ diameter, Shieldex silver plated polyamide fibres) and Spandex fibres (0.6, 1.2, and $1.7 \mathrm{~mm}$ diameter, Spandex Co. Ltd., China) were used to fabricate the twist-coil actuators. The torsional actuating samples were made by co-twisting the nylon 6 fibre with a slightly longer length of silver coated nylon multifilament fibre. The silver coated fibre was used for Joule heating of the twisted nylon fibre. A total of 428 twists per meter of fibre length were inserted with a constant axial stress 
of $10 \mathrm{MPa}$ applied to the nylon 6 monofilament during twist insertion. The twisted fibres were tethered from both ends and annealed at $120^{\circ} \mathrm{C}$ for 30 minutes [24]. The desired lengths of twisted nylon fibre and Spandex fibre were joined together using a metallic crimp to fabricate series actuator samples.

\subsection{Mechanical characterizations}

Tensile properties of the Spandex fibre samples were measured using a tensile tester (Shimadzu EZ-L Tester) and elastic moduli $(E)$ were calculated by fitting engineering stress $(\sigma)$ and extension ratio $(\lambda)$ data to the rubber elasticity theory [25]:

$\sigma=\frac{E}{3}\left(\lambda-\frac{1}{\lambda^{2}}\right)$

Torque-torsional stroke curves were generated experimentally by using the custom built torsion tester described previously [24] for different fibre diameters, where the slope of the curves denotes torsional stiffness. Fig. 3 shows the corresponding test apparatus along with the inset showing close-up of the individual components. Spandex fibres of different length were tested, since torsional stiffness proportionally depends on the fibre length. A force / distance transducer (Aurora Scientific lever arm model 305B) was utilised to apply constant torques to the Spandex fibre connected to the shaft of a near frictionless air bearing. During the test, the Spandex fibre was also subjected to a constant axial force from a second horizontally placed lever arm (Aurora Scientific model 300B) that allows linear contraction/expansion of the Spandex fibre. Gradually increasing torque applied by the lever arm to the shaft caused twisting of the Spandex at one end and therefore provided a measure of torque-torsional stroke relationship. Torsion testing of Spandex fibres in this manner was continued until coils formed and the torsional stiffnesses before and after coil formation could be determined. 

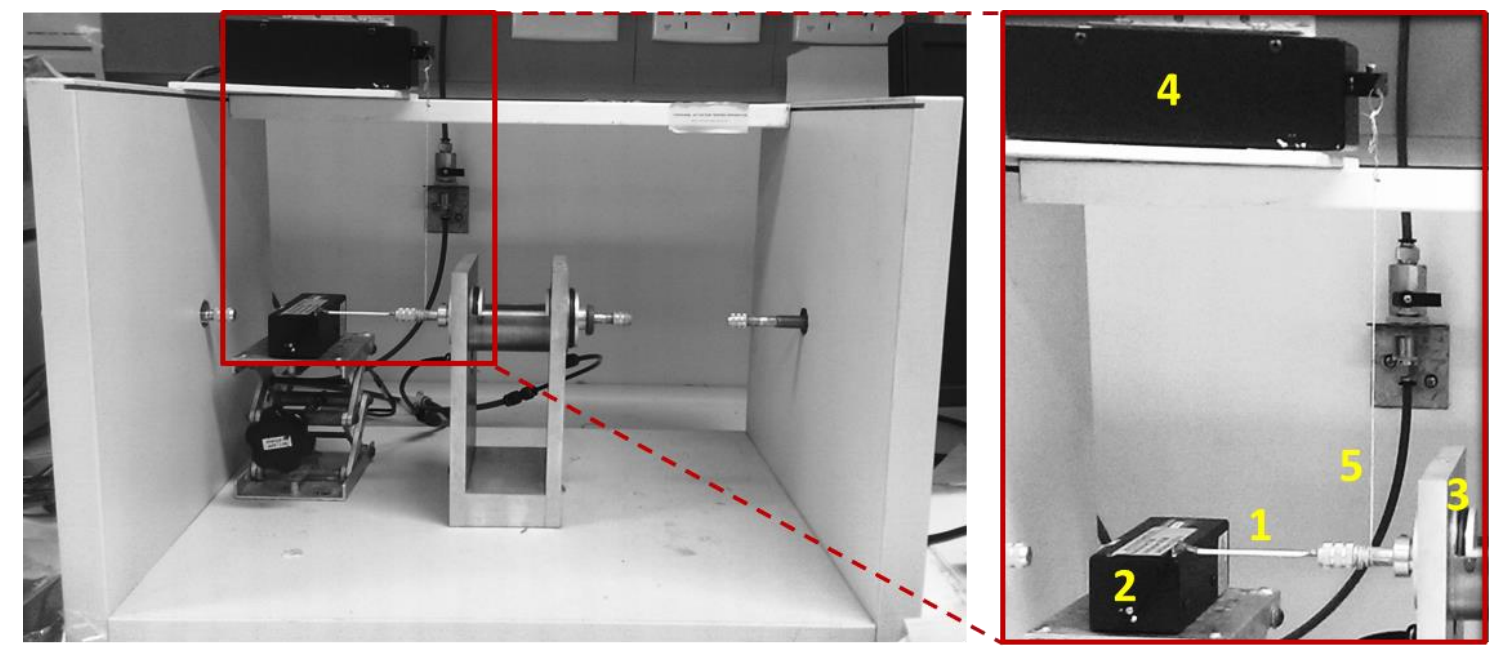

Fig. 3. Test apparatus for generating torque-torsional stroke curve (components are shown in right panel): (1) Spandex fibre, (2) first lever arm applying axial tension to the fibre, (3) air bearing for near frictionless fibre rotation, (4) second lever arm applying torque to the Spandex fibre, and (5) connecting fibre transferring torque from a second lever arm to the bearing shaft.

\subsection{Actuation tests}

The overall length change of the series actuator during electrical heating (over $26-125^{\circ} \mathrm{C}$ temperature range) of the nylon 6 torsional element was measured under isotonic conditions (Fig. 4). One end of the Spandex fibre was tethered to a force / distance transducer (Aurora Scientific lever arm 305B). The other end of the twisted nylon 6 fibre was firmly clamped, and a small constant tension was applied using the lever arm to keep the muscle straight. A DC power supply (TMS ${ }^{\circledR}$ Precision Variable 30V 5A DC Power Supply) was connected to the conductive element of the twisted nylon 6 fibre for Joule heating. To measure the temperature of the nylon 6 torsional actuator an infrared thermal imaging camera (MicroEpsilon TIM 160, supplied by Bestech Australia Pty Ltd) was used. Five different lengths of Spandex fibre were tested for each of the three samples having different diameters. The length of the torsional actuator fibre was varied according to the rotation requirements. Asprepared samples were used for the tests without changing geometrical configurations. 


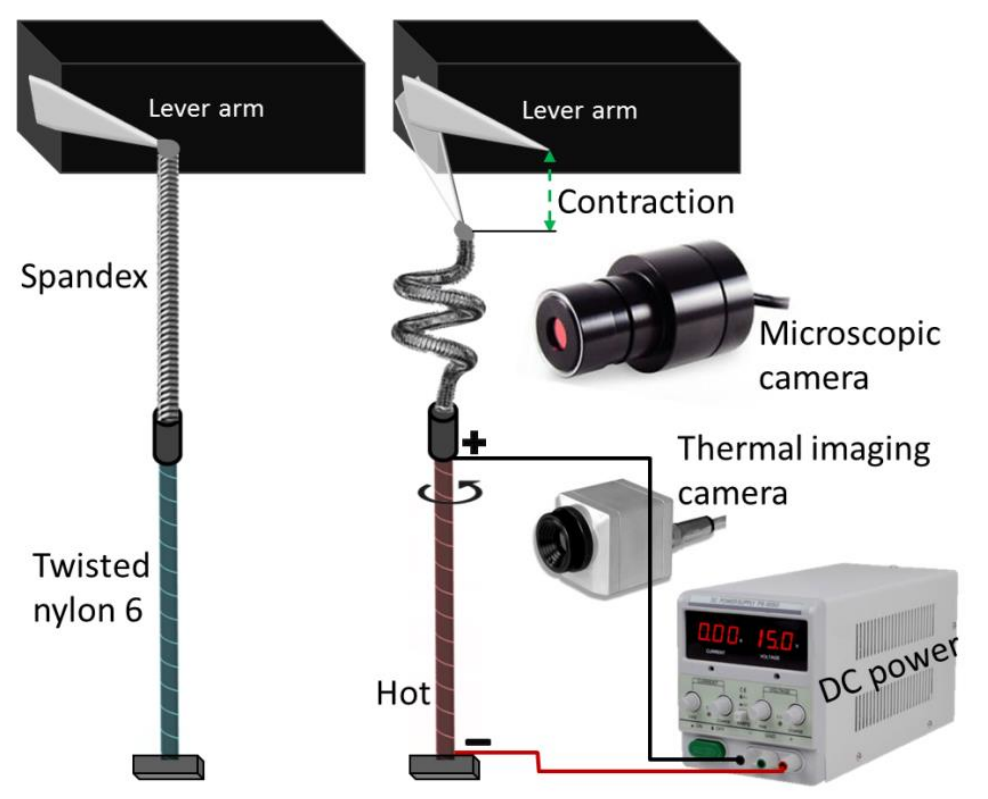

Fig. 4. Schematic illustration of actuation test set-up used for torsional actuation driven artificial muscle. The heat induced contraction due to twisting and coil formation in the Spandex fibre is schematically illustrated.

In a separate experiment, volume change of the nylon 6 fibres was measured optically over the experimental temperature range $\left(26-125^{\circ} \mathrm{C}\right)$ by heating with an infrared light. The diameter and bias angle of coils formed in the Spandex fibre were observed and measured microscopically (ISSCO-OPTEK microscopic camera) during the test.

\section{Results}

\subsection{Characteristic properties of individual actuator components}

The critical torques to initiate coil formation in the Spandex fibres were measured using the set-up shown in Fig. 3. Fig. 5(a) shows torque-torsional rotation curves for three different diameter of Spandex fibres each of $10 \mathrm{~mm}$ length. The axially applied force was kept constant at $0.02 \mathrm{~N}$ while the fibre was rotated at one end and fixed at the other end. All three samples showed a linear increase in torque with increasing rotation angle and with a distinct change in slope that corresponded to the formation of the first coil turn. The axial displacement of the fibre during twisting and with a constant axial force was also measured [Fig. 5(b)]. The fibre contraction during twisting and before coil formation was less than 3\% of the fibre length. By increasing the twists inserted, the length contraction increased significantly as the first coil turn formed. The larger diameter Spandex fibres generated the largest length contractions of up to $30 \%$ per each coil turn. 
The critical torque required to initiate coiling increased with Spandex fibre diameter. The predicted critical torques were calculated using the Ross criterion and the measured Spandex elastic modulus of $0.24 \mathrm{MPa}$. As shown in Fig. 5(c), the calculated critical torques increased with both Spandex fibre diameter and applied tensile force. The measured critical torques were slightly higher than the calculated values for all three fibres, but the results agree within an acceptable experimental error. Based on this comparison, it is concluded that the Ross criterion can be used as a reasonable estimate for the coiling torque of Spandex fibres in the further analysis of the series actuator.
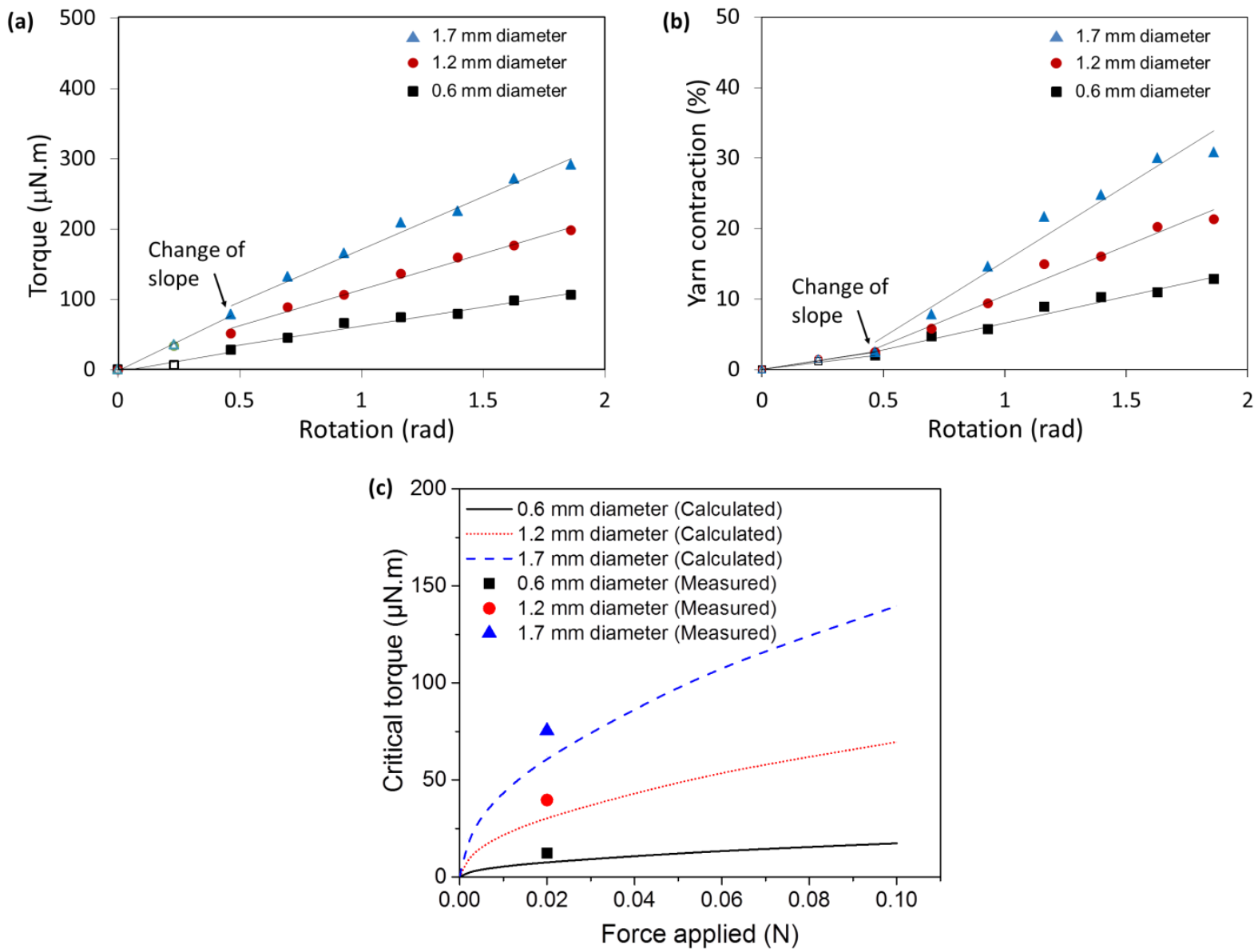

Fig. 5. Torque requirement for $10 \mathrm{~mm}$ long Spandex fibre; (a) torque-torsional rotation curves showing change in torsional stiffness of different diameter fibres, (b) fibre contraction during twisting and coiling, and (c) critical torques for differently loaded fibres.

Table 1 shows the parameters of the coil forming Spandex fibre that will be used for the theoretical calculation of actuator contraction. The amount of rotation needed to form the first coil turn (critical twist, $T_{\mathrm{c}}$ ) was calculated from the critical torque and the measured fibre 
stiffness, as given in equation (4). These values are independent of the length of the fibres and can be extended to Spandex fibres with various lengths.

Table 1. Spandex fibre (10 mm long) parameters for theoretical calculation of actuator contraction under $0.02 \mathrm{~N}$ axial force.

\begin{tabular}{|c|c|c|c|}
\hline $\begin{array}{c}\text { Spandex fibre } \\
\text { diameter }(D, \mathrm{~m})\end{array}$ & $\begin{array}{c}\text { Torsional modulus } \\
\text { before critical point } \\
\left(J_{\mathrm{N}} G_{\mathrm{N}}, \mu \mathrm{N} . \mathrm{m}^{2}\right)\end{array}$ & $\begin{array}{c}\text { Calculated critical } \\
\text { torque }\left(\tau_{\mathrm{c}}, \mu \mathrm{N} . \mathrm{m}\right)\end{array}$ & $\begin{array}{c}\text { Calculated critical } \\
\text { twist (turns } / \mathrm{m})\end{array}$ \\
\hline 0.0006 & 0.60 & 7.8 & 2.1 \\
\hline 0.0012 & 1.10 & 31.1 & 4.5 \\
\hline 0.0017 & 1.68 & 62.4 & 5.9 \\
\hline
\end{tabular}

The torsional rotation produced by thermally actuating a $0.54 \mathrm{~mm}$ diameter twisted nylon 6 fibre was calculated as previously described [20] using measured values of the radial thermal expansion and torsional stiffness of nylon 6. Free torsional rotation of twisted nylon 6 depends only on the amount of twist inserted into the fibre and the diameter expansion caused by the heating process [20]. The torsional stiffness of the heated nylon 6 fibre was calculated from the previously reported shear modulus $\left(G_{\mathrm{A}}\right)$ value at the maximum temperature of $125^{\circ} \mathrm{C}[24,26]$. Radial thermal expansion was measured microscopically over the same temperature range of $26-125^{\circ} \mathrm{C}$ that was used for actuation test (Fig. 6). These measurements were used to calculate the expected free rotation of the nylon 6 fibre, as given by equation (3). Since the diameter of the twisted nylon 6 fibre was kept constant for all experiments, the blocked torque was also independent of fibre length. Table 2 summarises the torsional and thermal properties of the twisted nylon 6 fibre used for theoretical calculation of muscle contraction. 


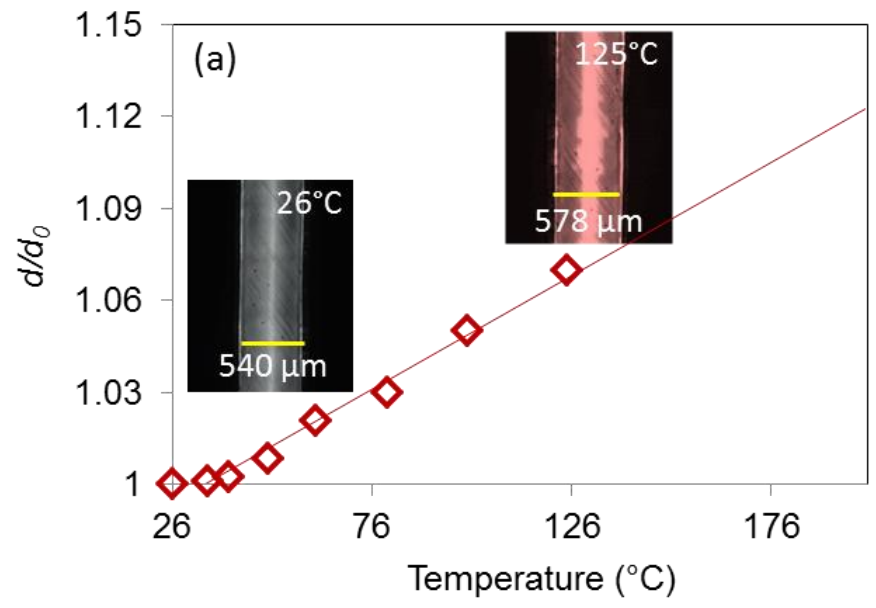

Fig. 6. Radial thermal expansion of twisted nylon 6 fibre $\left(\boldsymbol{d} / \boldsymbol{d}_{\mathbf{0}}\right)$ over $26-125^{\circ} \mathrm{C}$ temperature range.

Table 2. Torsional and thermal properties of $0.54 \mathrm{~mm}$ diameter twisted nylon fibre.

\begin{tabular}{|c|c|c|c|c|}
\hline Temperature & $\begin{array}{c}\text { Shear } \\
\text { modulus } \\
\left(G_{\mathrm{A}}, \mathrm{GPa}\right)\end{array}$ & $\begin{array}{c}\text { Radial thermal } \\
\text { expansion }(\%)\end{array}$ & $\begin{array}{c}\text { Blocked torque } \\
\left(\tau_{\text {blocked }}, \mu \mathrm{N} . \mathrm{m}\right)\end{array}$ & $\begin{array}{c}\text { Free rotation } \\
\left(\Delta T_{\text {free }},\right. \\
\text { turns } / \mathrm{m})\end{array}$ \\
\hline $26^{\circ} \mathrm{C}$ & 0.40 & - & - & - \\
\hline $125^{\circ} \mathrm{C}$ & 0.30 & $7 \%$ & 440 & 30 \\
\hline
\end{tabular}

\subsection{Spandex coil geometry}

The theoretical calculation of the muscle contraction requires the measurement of the diameter and bias angle of the coils that form in the Spandex fibre. Fig. 7(a) shows images of three different diameter Spandex fibres when the first coil is formed at a constant $0.02 \mathrm{~N}$ axial force. For all Spandex fibres investigated, the coil bias angles were the same and negative at this lowest applied force. Fig. 7(b) shows coils formed in the $1.7 \mathrm{~mm}$ diameter Spandex fibre when the axial force applied during coil formation varied from $0.02 \mathrm{~N}$ to 0.1 N. At higher axial forces, the coils were stretched out so that the coil bias angles gradually increased in a reversible process. 


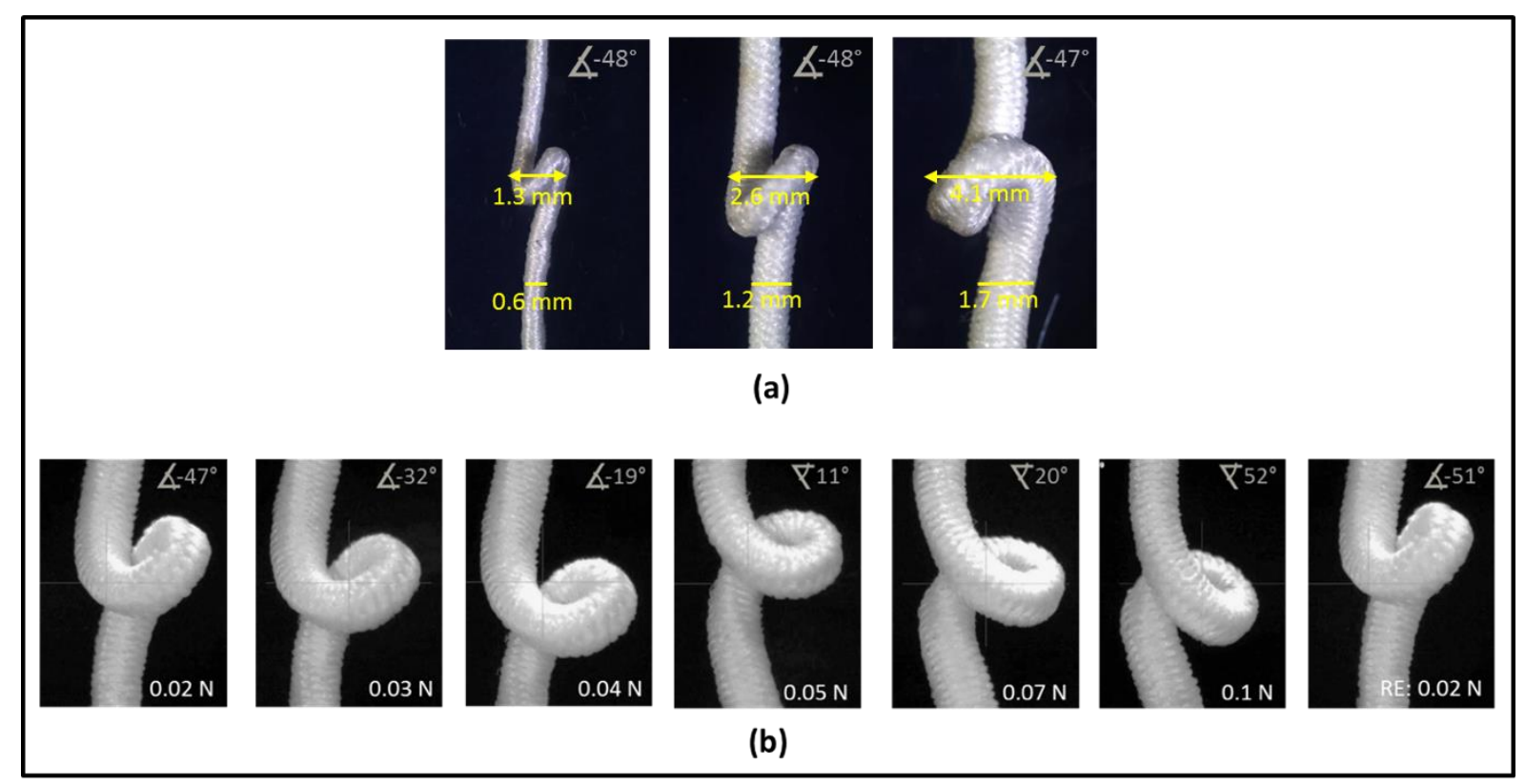

Fig. 7. Dimension and geometry of Spandex fibre coils; (a) three different diameter fibres showed similar coil bias angle when twisted under $0.02 \mathrm{~N}$ axial force, and (b) coils formed in $1.7 \mathrm{~mm}$ diameter fibre when actuated under different axial forces increasing from $0.02 \mathrm{~N}$ to $0.1 \mathrm{~N}$ and then repeated at $0.02 \mathrm{~N}$.

To calculate the overall actuation of twist-coil actuator from equations (1) and (7), the active diameter of coils must be estimated. The top-view of a single coil is illustrated in Fig. 8(a), highlighting the active diameter of the coil $\left(D_{\mathrm{c}}\right)$ as the subtraction of fibre diameter $\left(D_{\mathrm{y}}\right)$ from coil outer diameter $(D)$. The validity of this assumption was experimentally evaluated by using a solid rubber cylinder. A portion of the solid rubber cylinder (length, $L_{\mathrm{S}}=264 \mathrm{~mm}$ and diameter, $D_{\mathrm{y}}=10 \mathrm{~mm}$ ) was wrapped around a $10 \mathrm{~mm}$ diameter metal mandrel generating 4 full coil turns [Fig. 8(b)]. The outer diameter $(D)$ of the coil was measured to be $30.5 \mathrm{~mm}$; therefore, the active coil diameter was calculated as $20.5 \mathrm{~mm}$ [Fig. 8(c)]. Following the openup helix model shown in Fig. 8(c), the length of coiled rubber can be expressed as:

$N_{\mathrm{c}} L_{\mathrm{c}}=\sqrt{\left(N_{\mathrm{c}} L_{\mathrm{S}}\right)^{2}-\left(\pi \cdot N_{\mathrm{c}} \cdot D_{\mathrm{c}}\right)^{2}}$

The calculated coil length was found to be $\sim 58 \mathrm{~mm}$ which agrees quite accurately with the measured coil length of $\sim 61 \mathrm{~mm}$. These results confirm that the active coil diameter is the most appropriate dimension in using the helix model to evaluate the overall contraction of the series muscle. 


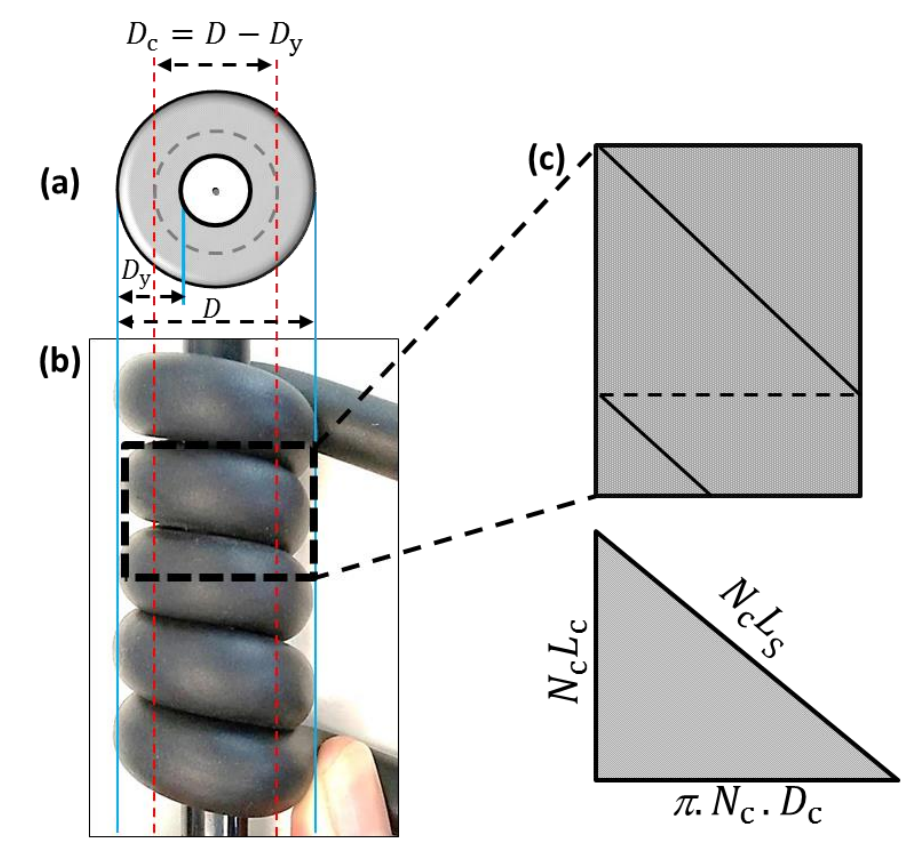

Fig. 8. Experimental validation of the use of active coil diameter $\left(\boldsymbol{D}_{\mathbf{c}}\right)$ to calculate overall series muscle contraction; (a) coil dimensions, (b) actual image of the mandrel coil and (c) single helix model for calculation.

\subsection{Tensile actuation}

The actuation of twist-coil actuators with different geometries was measured using the set-up shown in Fig. 4. First, the effect of Spandex fibre length on the actuation strains was explored by systematically increasing the length of Spandex $(1.7 \mathrm{~mm}$ diameter) while the Spandex lengths ratio to nylon 6 fibre $(0.54 \mathrm{~mm}$ diameter) was kept constant at 1:2. Reproducible results with fully reversible actuation without hysteresis were obtained for all samples when heated and cooled over the $26-125^{\circ} \mathrm{C}$ temperature range [Fig. 9]. Nearly identical actuation strains of $\sim 10 \%$ based on the total summed length of nylon 6 fibre and Spandex fibres were observed for all samples regardless of Spandex fibre length. This actuation strain is similar to that obtained from fully twisted and coiled polymer fibres after multiple cycles of "training" when heated over the same temperature range, Table 3 [8]. Peak contraction over the 26$125^{\circ} \mathrm{C}$ temperature was achieved as fast as 50 seconds through Joule heating. The cooling process, however, took around 10 minutes when the electrical power was turned off. A faster cooling of 2 minutes was obtained in an active air-cooling environment. The contraction rate of the twist-coil actuators was higher when the samples were heated above $60^{\circ} \mathrm{C}$ which accords with larger torsional rotation of twisted nylon 6 at temperatures above its glass transition temperature $\left(T_{\mathrm{g}} \sim 50^{\circ} \mathrm{C}\right)[27]$. 


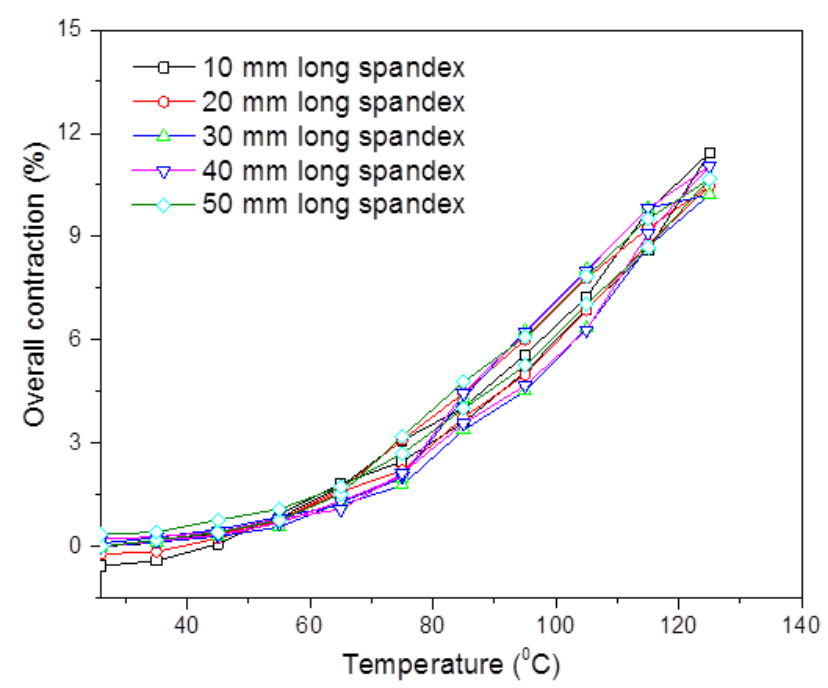

Fig. 9. Experimental results for contraction of a series of twist-coil actuators with varying Spandex length at a constant Spandex to nylon 6 length ratio of 1:2.

Table 3. Thermal actuation performance of twisted and coiled polymer fibres.

\begin{tabular}{|c|c|c|}
\hline Material & $\begin{array}{c}\text { Temperature } \\
\text { Range }\end{array}$ & $\begin{array}{c}\text { Tensile } \\
\text { Stroke }\end{array}$ \\
\hline Polyethylene braided yarn & $25-120^{\circ} \mathrm{C}$ & $13 \%$ \\
\hline Nylon-6,6 multifilament yarn & $25-120^{\circ} \mathrm{C}$ & $8 \%$ \\
\hline Nylon-6 monofilament & $25-120^{\circ} \mathrm{C}$ & $10 \%$ \\
\hline Nylon-6,6 monofilament & $25-120^{\circ} \mathrm{C}$ & $10 \%$ \\
\hline
\end{tabular}

\section{Discussion}

Calculated tensile strokes occurring as a result of torsion-induced coil formation in the Spandex fibre were obtained using equations (1), (2), (3), (4), (5) and (7). The free torsional stroke expected from the nylon fibre was first calculated using equation (3) from the measured nylon fibre diameter at various temperatures over the experimental range. Measured fibre torsional stiffnesses were then used with equation (5) to determine the torsional stroke available when the nylon fibre was connected in series with the Spandex fibre. This calculated reduced torsional stroke $(\Delta T)$ was experimentally verified by observing the junction rotation during the heating of a $180 \mathrm{~mm}$ long nylon 6 fibre attached in series to a $30 \mathrm{~mm}$ long, $0.6 \mathrm{~mm}$ diameter Spandex fibre [Fig. 10(a)]. At the maximum actuation temperature $\left(125^{\circ} \mathrm{C}\right)$, a measured rotation of 2 turns was observed which agreed perfectly with the calculated result. The critical torsional stroke needed for first coil turn formation was 
calculated using equations (2) and (3) and the measured Spandex fibre torsional modulus. The analysis of coiling can be expressed graphically by plotting the torque-stroke curve of the twisted nylon fibre super-imposed with the torsional stiffness curve of the coiling Spandex fibres. Fig. 10(b) shows the torque-stroke curve in which free rotation and blocked torque of a $20 \mathrm{~mm}$ long twisted nylon fibre is plotted and connected (green dotted line). The green shaded region now shows the rotation available $\left(l_{\mathrm{A}} \Delta T-l_{\mathrm{N}} T_{\mathrm{c}}\right)$ for Spandex coil formation.

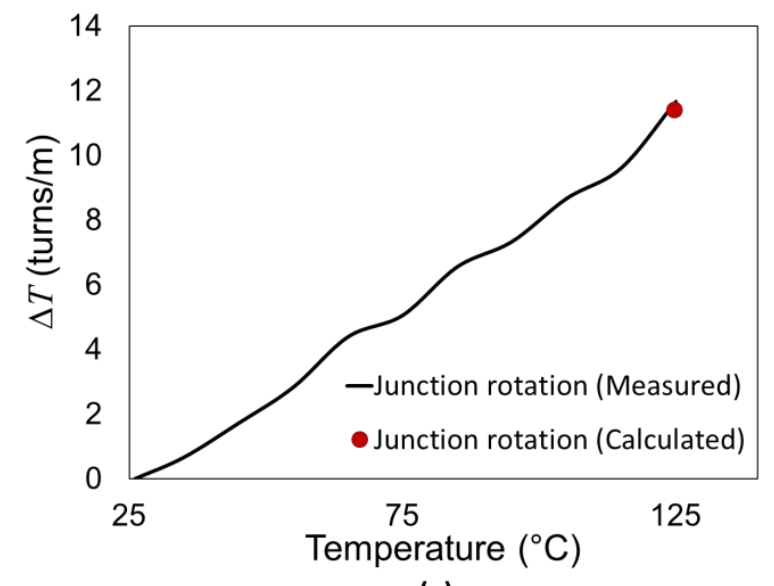

(a)

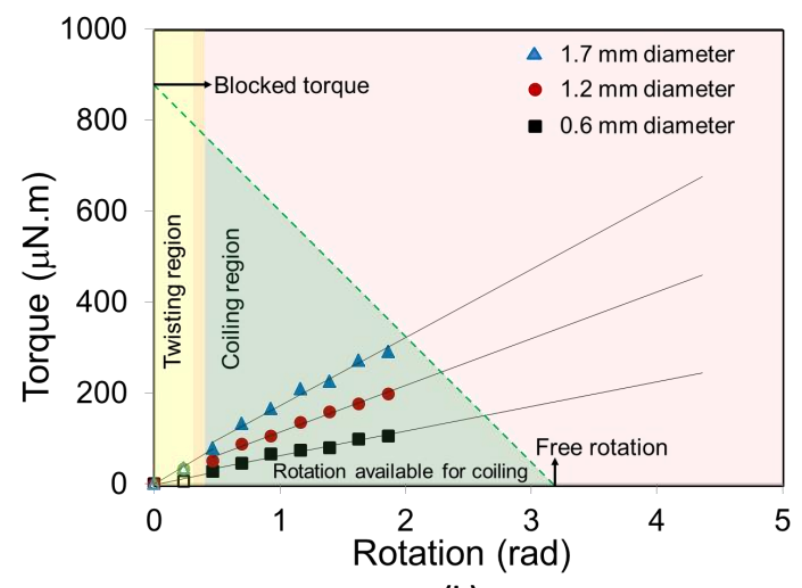

(b)

Fig. 10. (a) Thermally induced torsional rotation at the junction of a $180 \mathrm{~mm}$ long nylon 6 fibre and $30 \mathrm{~mm}$ long, $0.6 \mathrm{~mm}$ diameter Spandex fibre. (b) Graphical representation of effective torsional rotation of a $20 \mathrm{~mm}$ long $540 \mu \mathrm{m}$ twisted nylon 6 fibre when heated from $26^{\circ} \mathrm{C}$ to $125^{\circ} \mathrm{C}$ and the formation of coils in serially attached $10 \mathrm{~mm}$ long Spandex fibres of three different diameters, as indicated.

Finally, the tensile stroke was calculated from equation (7) using the measured coil diameter and bias angles. Fig. 11(a-c) shows plots for calculated muscle contraction as a function of nylon 6 fibre length, using three different Spandex diameters each at five different lengths. Tensile contractions are expressed as a percentage with respect to the total initial length of twist-coil actuators. All Spandex fibres showed a peak contraction as the twisted nylon 6 fibre length increased. The peaks in contraction strain are due to competition between increasing free stroke and decreasing torsional stiffness as the nylon 6 fibre length increases. The maximum contraction strain was predicted when the lengths ratio of Spandex fibre to twisted nylon 6 fibre reaches $\sim 1: 2$ [ Fig. 11(d)]. The maximum peak contraction was found to depend only on the Spandex fibre diameter regardless of the Spandex length. The largest diameter fibre (1.7 mm diameter) was estimated to generate a maximum peak contraction of $\sim 12 \%$ compared to $\sim 10 \%$ for fibres with $1.2 \mathrm{~mm}$ diameter and $\sim 7 \%$ for $0.6 \mathrm{~mm}$ diameter 
fibres. This trend reflects the dominating contribution of the fibre diameter which results in the formation of longer coil turns of Spandex.
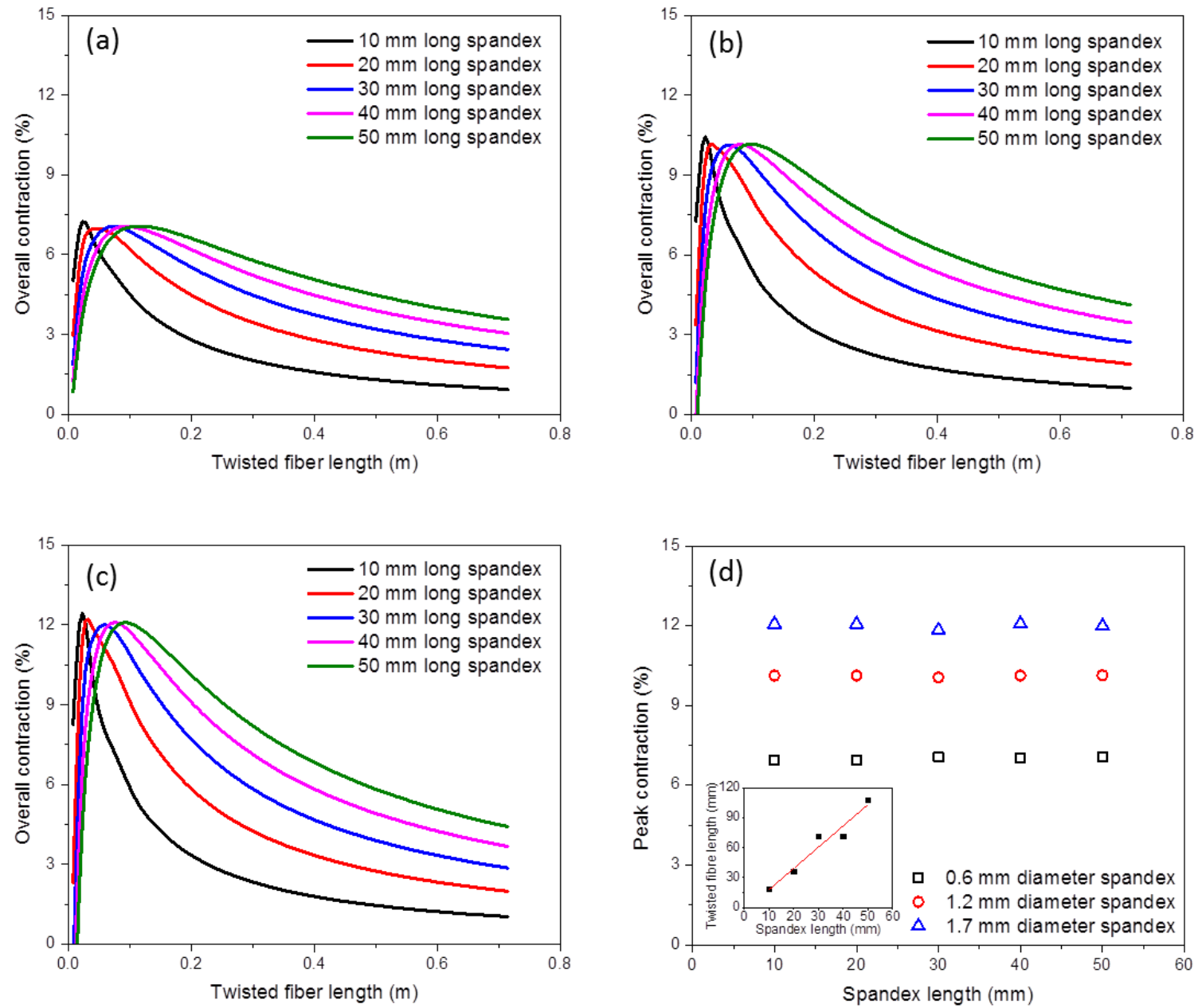

Fig. 11. Theoretically predicted contraction of Spandex fibre attached to a twisted nylon 6 fibre torsional artificial muscle in terms of different muscle lengths for different diameter Spandex fibres: (a) $0.6 \mathrm{~mm}$, (b) $1.2 \mathrm{~mm}$ diameter fibre, (c) $1.7 \mathrm{~mm}$ diameter fibre; and (d) peak contraction comparison (inset shows the ratio of twisted fibre to Spandex fibre that provides the peak contraction).

The theoretically calculated results were then compared to the measured muscle contraction. Fig. 12(a) shows good agreement between calculated and measured peak actuations for actuators with the optimised 2:1 lengths ratio of twisted nylon 6 fibre to Spandex fibre. Actuation tests were also conducted for a $10 \mathrm{~mm}$ long Spandex fibre attached with $20 \mathrm{~mm}$ twisted nylon 6 fibre under different isotonic loading conditions [Fig. 12(b)]. As presented in Fig. 5(c) the critical torque and critical rotation are larger for muscles subjected to higher axial force. A lower fraction of the overall twisted fibre rotation is then available for coiling 
the Spandex fibre. Consequently, the overall muscle contraction decreased significantly at higher applied tensile forces. Notably, fully reversible actuation without any permanent creep was observed even at the higher loading conditions. For all of the experimental conditions, experimental results agreed with the theoretical calculations within the limit of $\sim 90 \%$ accuracy.
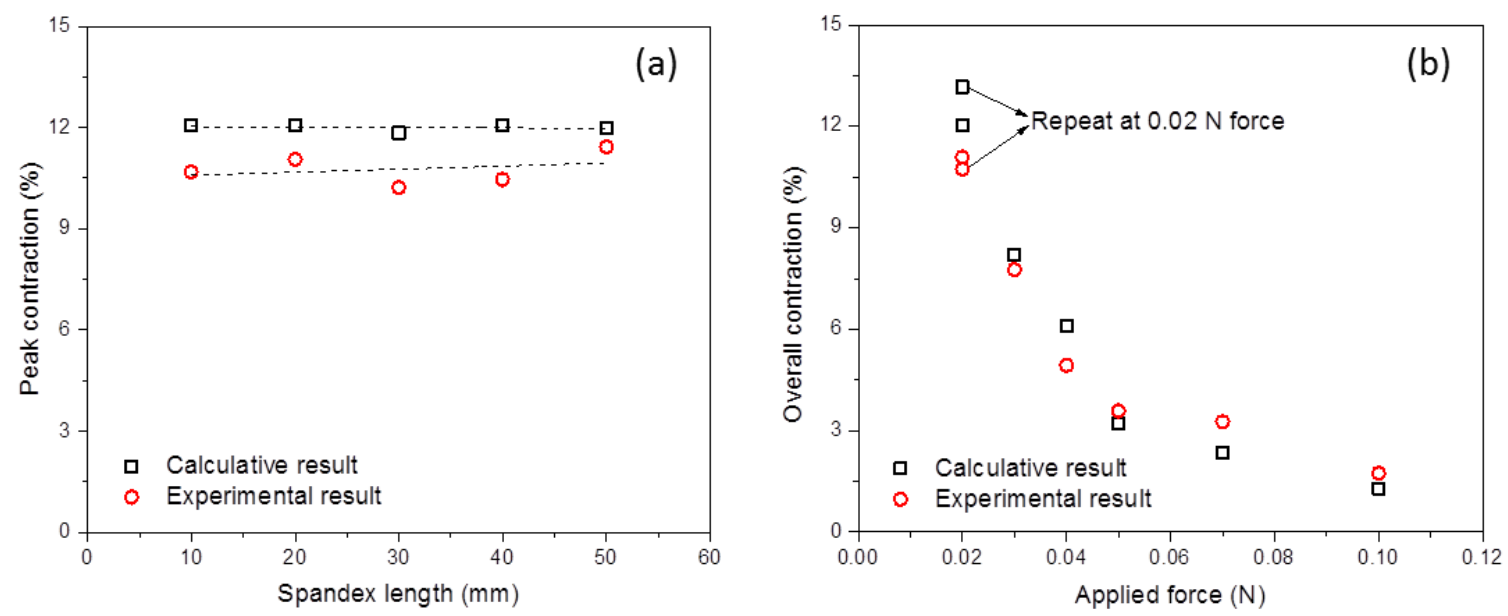

Fig. 12. Comparison of theoretically calculated muscle contraction to measured values: (a) as a function of Spandex length, and (b) under different axial force.

Previous studies have shown that over-twisted and coiled polymer fibre actuators exhibit low structural stability during the first few heating / cooling actuation cycles [20, 24, 28]. Consequently, training of the fibres is necessary before a consistent actuation is achieved. This criterion also exists when the actuation experiments are conducted under different loading conditions [20]. In this work, highly reversible actuation was obtained from the very first cycle due to the presence of the highly elastic Spandex fibre that acts as a return spring mechanism. Coils of Spandex fibre store a significant amount of torque while formed, and the release of this torque helps the retwisting of twisted fibre during the cooling process. Therefore, the overall number of twists that is available in the nylon 6 fibre to generate the next coiling upon heating remains unchanged over consecutive actuation cycles.

\section{Conclusions}

A new type of tensile artificial muscle is demonstrated herein with a combination of torsional and tensile actuation. A set of identical twisted nylon 6 fibres was fabricated having $540 \mu \mathrm{m}$ diameter and $\sim 28$ twists per meter of fibre length. Spandex fibre was attached to the twisted fibre in series under fixed tension so that the ends were blocked from torsional rotation but 
allowing tensile movement. Thermally induced torsional actuation of the twisted fibre caused rotation at the junction of this fibre and the Spandex fibre. The subsequent formation of coils in the Spandex fibre resulted in overall muscle length contraction. Torsional rotation of twisted fibre and resultant Spandex coil geometry are utilized to theoretically predict the amount of muscle contraction by means of a modified single helix theory. Good agreement was found in between measured and calculated results. Practical applicability of this muscle was evaluated by using different mechanical loading conditions. The actuation strain was increased when larger diameter Spandex fibres were used. It was also found that fully reversible actuation was achieved without any training cycles.

\section{Acknowledgments}

The authors thank the Australian Research Council for supporting this work through the Professorial Fellowship (GMS DP110101073), Centre of Excellence (CE 140100012), and Discovery Early Career Researcher Award (JF DE12010517).

\section{References}

[1] M. Shahinpoor, K.J. Kim, M. Mojarrad, Artificial Muscles: Applications of Advanced Polymeric Nanocomposites: CRC Press; 2007.

[2] J. Foroughi, G.M. Spinks, G.G. Wallace, J. Oh, M.E. Kozlov, S. Fang, et al., Torsional Carbon Nanotube Artificial Muscles, Science, 334(2011) 494-7.

[3] M.D. Lima, N. Li, M. Jung de Andrade, S. Fang, J. Oh, G.M. Spinks, et al., Electrically, Chemically, and Photonically Powered Torsional and Tensile Actuation of Hybrid Carbon Nanotube Yarn Muscles, Science, 338(2012) 928-32.

[4] S.M. Mirvakili, A. Pazukha, W. Sikkema, C.W. Sinclair, G.M. Spinks, R.H. Baughman, et al., Niobium Nanowire Yarns and their Application as Artificial Muscles, Advanced Functional Materials, 23(2013) 4311-6.

[5] Z. Fan, K. Raun, L. Hein, H.-E. Kiil, Application of Artificial Muscles as Actuators in Engineering Design, in: X.-T. Yan, C. Jiang, B. Eynard (Eds.), Advanced Design and Manufacture to Gain a Competitive Edge: New Manufacturing Techniques and their Role in Improving Enterprise Performance, Springer London, London, 2008, pp. 875-84.

[6] G. Han, G. Shi, Conducting polymer electrochemical actuator made of high-strength three-layered composite films of polythiophene and polypyrrole, Sensors and Actuators B: Chemical, 99(2004) 525-31.

[7] P. Du, X. Lin, X. Zhang, A multilayer bending model for conducting polymer actuators, Sensors and Actuators A: Physical, 163(2010) 240-6.

[8] C.S. Haines, M.D. Lima, N. Li, G.M. Spinks, J. Foroughi, J.D.W. Madden, et al., Artificial Muscles from Fishing Line and Sewing Thread, Science, 343(2014) 868-72.

[9] J. Foroughi, G.M. Spinks, S. Aziz, A. Mirabedini, A. Jeiranikhameneh, G.G. Wallace, et al., Knitted Carbon-Nanotube-Sheath/Spandex-Core Elastomeric Yarns for Artificial Muscles and Strain Sensing, ACS Nano, (2016). 
[10] S.H. Kim, M.D. Lima, M.E. Kozlov, C.S. Haines, G.M. Spinks, S. Aziz, et al., Harvesting temperature fluctuations as electrical energy using torsional and tensile polymer muscles, Energy \& Environmental Science, 8(2015) 3336-44.

[11] C.H. Kwon, K.-Y. Chun, S.H. Kim, J.-H. Lee, J.-H. Kim, M.D. Lima, et al., Torsional behaviors of polymer-infiltrated carbon nanotube yarn muscles studied with atomic force microscopy, Nanoscale, 7(2015) 2489-96.

[12] R. Bogue, Artificial muscles and soft gripping: a review of technologies and applications, Industrial Robot: An International Journal, 39(2012) 535-40.

[13] C.-H. Li, C. Wang, C. Keplinger, J.-L. Zuo, L. Jin, Y. Sun, et al., A highly stretchable autonomous self-healing elastomer, Nat Chem, 8(2016) 618-24.

[14] Y. Bar-Cohen, Electroactive Polymer (EAP) Actuators as Artificial Muscles: Reality, Potential, and Challenges: Society of Photo Optical; 2004.

[15] K.-Y. Chun, S. Hyeong Kim, M. Kyoon Shin, C. Hoon Kwon, J. Park, Y. Tae Kim, et al., Hybrid carbon nanotube yarn artificial muscle inspired by spider dragline silk, Nat Commun, 5(2014).

[16] C.S. Haines, N. Li, G.M. Spinks, A.E. Aliev, J. Di, R.H. Baughman, New twist on artificial muscles, Proceedings of the National Academy of Sciences, 113(2016) 11709-16.

[17] N. Li, C.S. HAINES, M.D. LIMA, D.A.M. Jung, S. Fang, J. Oh, et al., Coiled and noncoiled twisted nanofiber yarn and polymer fiber torsional and tensile actuators, Google Patents2014.

[18] S.M. Mirvakili, A. Rafie Ravandi, I.W. Hunter, C.S. Haines, N. Li, J. Foroughi, et al., Simple and strong: twisted silver painted nylon artificial muscle actuated by Joule heating, 2014, pp. 90560I-I-10.

[19] G.M. Spinks, Stretchable artificial muscles from coiled polymer fibers, Journal of Materials Research, 31(2016) 2917-27.

[20] S. Aziz, S. Naficy, J. Foroughi, H.R. Brown, G.M. Spinks, Controlled and scalable torsional actuation of twisted nylon 6 fiber, Journal of Polymer Science Part B: Polymer Physics, 54(2016) 1278-86.

[21] S. Goyal, N.C. Perkins, C.L. Lee, Nonlinear dynamics and loop formation in Kirchhoff rods with implications to the mechanics of DNA and cables, Journal of Computational Physics, 209(2005) 371-89.

[22] V.G.A. Goss, G.H.M. van der Heijden, J.M.T. Thompson, S. Neukirch, Experiments on snap buckling, hysteresis and loop formation in twisted rods, Experimental Mechanics, 45(2005) 101.

[23] A.L. Ross, Cable Kinking Analysis and Prevention, Journal of Engineering for Industry, 99(1977) 112-5.

[24] S. Aziz, S. Naficy, J. Foroughi, H.R. Brown, G.M. Spinks, Characterisation of torsional actuation in highly twisted yarns and fibres, Polymer Testing, 46(2015) 88-97.

[25] H. Xin, S.Z. Saricilar, H.R. Brown, P.G. Whitten, G.M. Spinks, Effect of First Network Topology on the Toughness of Double Network Hydrogels, Macromolecules, 46(2013) 661320.

[26] T. Shibukawa, V.D. Gupta, R. Turner, J.H. Dillon, A.V. Tobolsky, Temperature Dependence of Shear Modulus and Density of Nylon-6, Textile Research Journal, 32(1962) 1011-2.

[27] J.E. McIntyre, T. Institute, Synthetic Fibres: Nylon, Polyester, Acrylic, Polyolefin: CRC Press; 2005.

[28] S. Aziz, S. Naficy, J. Foroughi, H.R. Brown, G.M. Spinks, Thermomechanical effects in the torsional actuation of twisted nylon 6 fiber, Journal of Applied Polymer Science, 134(2017) 45529-n/a. 
